\title{
The epitrigeminal approach to the brainstem
}

\author{
Georgios Andrea Zenonos, MD, David Fernandes-Cabral, MD, Maximiliano Nunez, MD, \\ Stefan Lieber, MD, Juan Carlos Fernandez-Miranda, MD, and Robert Max Friedlander, MD, MA
}

Department of Neurological Surgery, University of Pittsburgh Medical Center, Pittsburgh, Pennsylvania

\begin{abstract}
OBJECTIVE Surgical approaches to the ventrolateral pons pose a significant challenge. In this report, the authors describe a safe entry zone to the brainstem located just above the trigeminal entry zone which they refer to as the "epitrigeminal entry zone."
\end{abstract}

METHODS The approach is presented in the context of an illustrative case of a cavernous malformation and is compared with the other commonly described approaches to the ventrolateral pons. The anatomical nuances were analyzed in detail with the aid of surgical images and video, anatomical dissections, and high-definition fiber tractography (HDFT). In addition, using the HDFT maps obtained in 77 normal subjects (154 sides), the authors performed a detailed anatomical study of the surgically relevant distances between the trigeminal entry zone and the corticospinal tracts.

RESULTS The patient treated with this approach had a complete resection of his cavernous malformation, and improvement of his symptoms. With regard to the HDFT anatomical study, the average direct distance of the corticospinal tracts from the trigeminal entry zone was $12.6 \mathrm{~mm}$ (range $8.7-17 \mathrm{~mm}$ ). The average vertical distance was $3.6 \mathrm{~mm}$ (range -2.3 to $8.7 \mathrm{~mm}$ ). The mean distances did not differ significantly from side to side, or across any of the groups studied (righthanded, left-handed, and ambidextrous).

CONCLUSIONS The epitrigeminal entry zone to the brainstem appears to be safe and effective for treating intrinsic ventrolateral pontine pathological entities. A possible advantage of this approach is increased versatility in the rostrocaudal axis, providing access both above and below the trigeminal nerve. Familiarity with the subtemporal transtentorial approach, and the reliable surgical landmark of the trigeminal entry zone, should make this a straightforward approach. https://thejns.org/doi/abs/10.3171/2016.12.JNS162561

KEY WORDS brainstem approaches; cavernous malformations; epitrigeminal approach; intrinsic brainstem lesions; safe entry zone; subtemporal transtentorial approach; surgical technique

L ESIONS of the ventrolateral pons are particularly challenging to access. Multiple approaches have been described in the literature, each one with its own indications, merits, and shortcomings. , $^{4,10,11,15,17}$ These approaches are often planned according to 2 points: 1) the epicenter of the lesion, and 2) either a point where the lesion presents to a pial surface, or by the closest "safe entry zone" to the brainstem. These safe entry zones are defined as corridors that are least likely to disrupt eloquent structures, and thus are the least likely to result in neurological deficits when transgressed. ${ }^{4,5,10,11,15,17}$

In this article we describe in detail an approach to the ventrolateral pons through a safe entry zone that, to our knowledge, has not previously been described. This safe entry zone is located just rostral to the posteriormost point of the trigeminal nerve entry zone in the pons, and is accessed through a subtemporal transtentorial surgical corridor. To avoid confusion with previous reports, we refer to this as the "epitrigeminal approach." The approach is presented in the context of an illustrative case of a brainstem cavernous malformation. We analyze the surgical nuances of this technique, and compare the epitrigeminal approach to other described approaches to the ventrolateral pons. Furthermore, we discuss in detail the relevant anatomy, and investigate the variability of crucial anatomical relationships with high-definition fiber tractography (HDFT).

\section{Methods}

Seventy-seven normal subjects were analyzed with HDFT to determine the relationship of the corticospinal tract (CST) to the epitrigeminal safe entry zone.

\section{The Human Connectome Project}

We performed HDFT on 77 individual diffusion scans that were randomly selected from the Human Connec-

ABBREVIATIONS CN = cranial nerve; $\mathrm{CST}=$ corticospinal tract; $\mathrm{HCP}=$ Human Connectome Project; HDFT = high-definition fiber tractography.

SUBMITTED October 7, 2016. ACCEPTED December 21, 2016.

INCLUDE WHEN CITING Published online August 25, 2017; DOI: 10.3171/2016.12.JNS162561. 


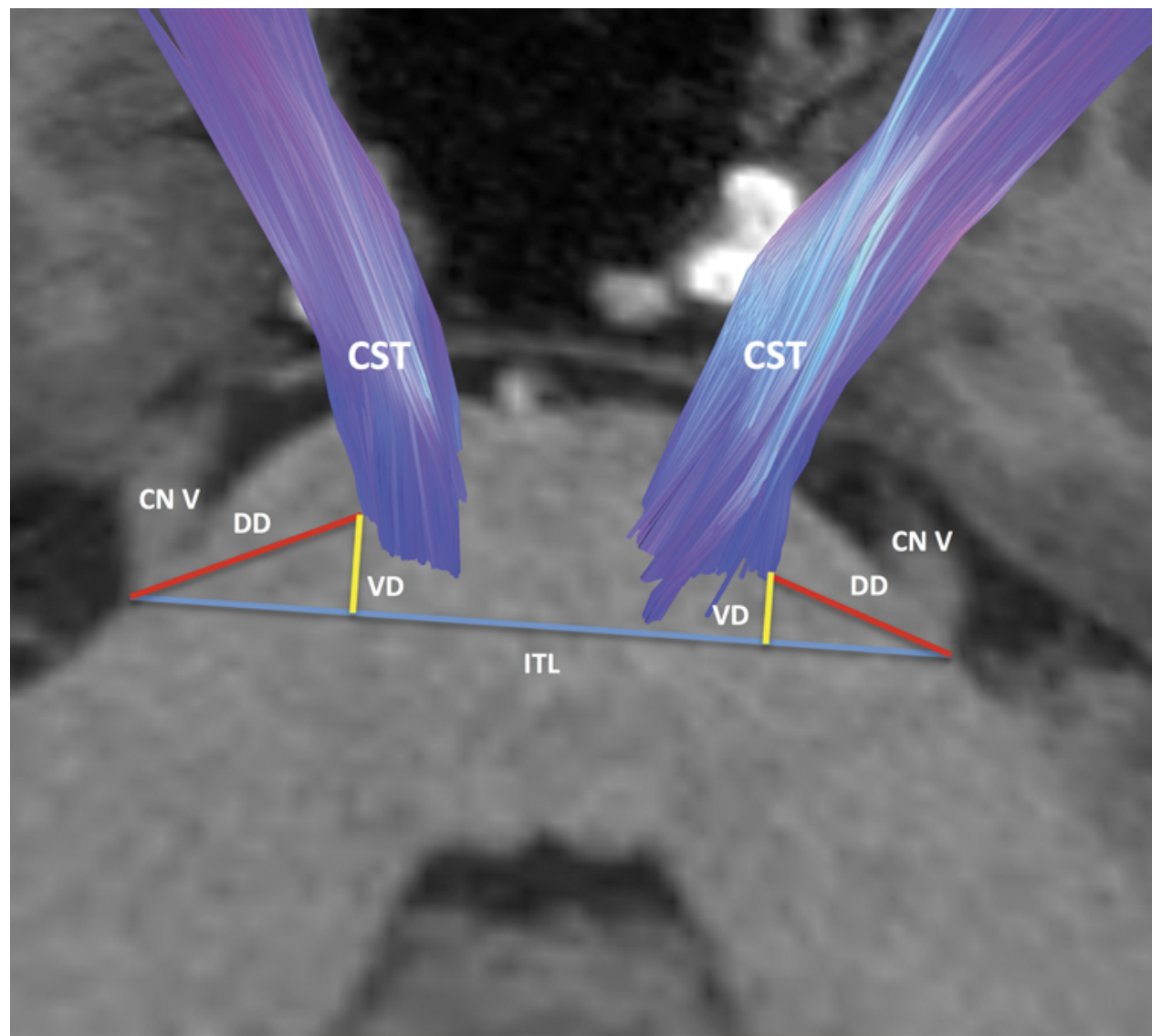

FIG. 1. Surgically relevant measurements of the CSTs from the epitrigeminal safe entry zone. An axial image at a level close to the rostral end of the trigeminal entry zone approximates the level of the epitrigeminal zone. $D D=$ direct distance of the posterolateral corticospinal fibers from the epitrigeminal zone; ITL = intertrigeminal line extending across the epitrigeminal zones on either side; VD = vertical distance - the distance of the posterolateral CSTs from the intertrigeminal line.

tome Project (HCP) (35 men, 42 women; subject-based approach). The diffusion scans are made freely available online by the HCP. ${ }^{16}$ The mean age of the subjects was 29 years (range $22-36$ years). Sixty-one $(79.2 \%)$ subjects were right-handed, $10(13 \%)$ were left-handed, and 6 (7.8\%) were ambidextrous.

\section{Image Acquisition and Reconstruction}

The HCP diffusion data were acquired in a Siemens 3-T Skyra scanner using a 2D spin-echo single-shot multiband echo planar imaging sequence, with a multiband factor of 3 and monopolar gradient pulse. ${ }^{16}$ The special resolution was $1.25 \mathrm{~mm}$ isotropic, TR $5500 \mathrm{msec}$, TE $89 \mathrm{msec}$. A multishell diffusion scheme was used. The b-values were 1000, 2000, and 3000 seconds $/ \mathrm{mm}^{2}$. All diffusion data were acquired by the Washington University-University of Minnesota (WU-Minn) HCP consortium. The diffusion data were reconstructed at the University of Pittsburgh using a generalized q-sampling imaging method to model an orientation distribution function in each brain voxel. ${ }^{19}$

\section{Fiber Tracking}

Whole-brain fiber tracking was conducted at the University of Pittsburgh High-Definition Fiber Tractography Laboratory with the aid of a deterministic fiber-tracking algorithm (DSI Studio; dsi-studio.labsolver.org). ${ }^{18}$ The fiber tracts of the middle cerebellar peduncle; 8,13 cranial nerves (CNs) III, V, VII, and VIII; ${ }^{20}$ the medial lemnisci, medial longitudinal fascicles, and tectospinal tracts; ${ }^{8,13}$ as well as the CSTs, ${ }^{7,13}$ were reconstructed similarly to those described in prior reports. Fiber tracking was initiated from the whole-brain seed by using all orientations present within a voxel until 100,000 streamlines were detected. To maximize the detection of studied tracts, the following parameters were kept constant across the scans: maximum turning angle of $60^{\circ}$, step size of $0.5 \mathrm{~mm}$, smoothing 0.8 , and a length constraint of 100-200 mm. All fibers terminating outside the trajectory of the involved tracts were deleted.

\section{Anatomical Measurements}

The anatomical measurements obtained in this study and the measurement methodology are explained in Fig. 1. Given that the size and extent of the CSTs on each side could differ depending on whether someone is right-handed, left-handed, or ambidextrous, the left and right sides were evaluated separately in each group.

\section{Statistical Analysis}

The statistical analyses were performed using the Mi- 

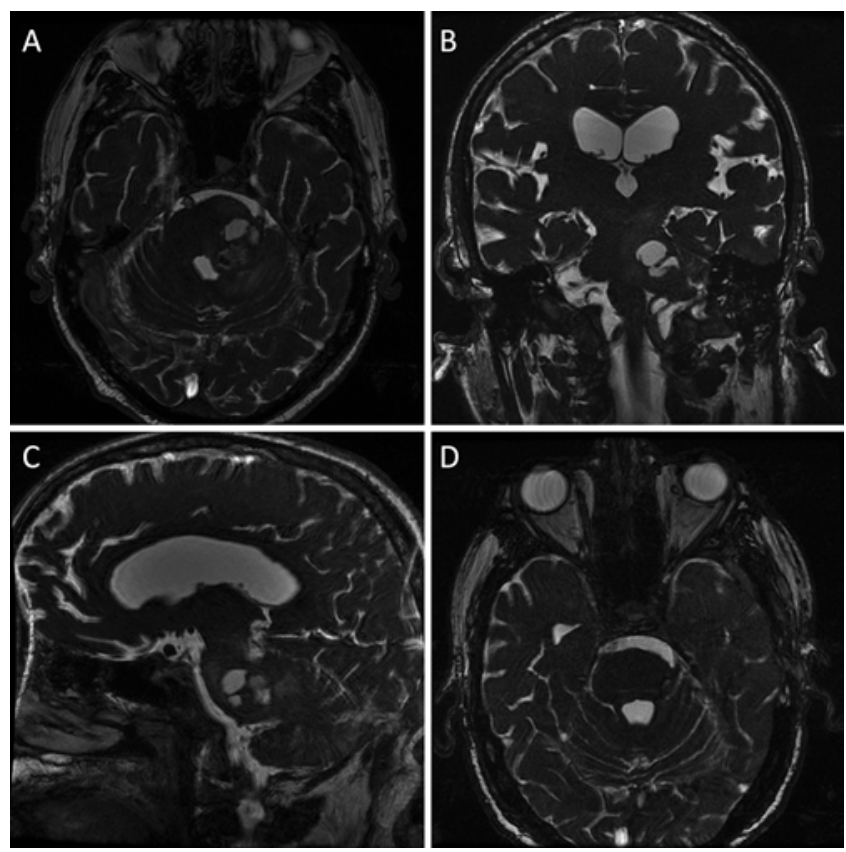

FIG. 2. Preoperative and postoperative MR imaging. A-C: Preoperative axial, coronal, and sagittal T2-weighted images, respectively, of the cavernous malformation. An 11.2- $\mathrm{cm}^{3}$ multiloculated hemorrhagic mass spanned the entire left ventrolateral pons, extending both above and below the level of the trigeminal nerve, down to the pontomedullary fissure. D: Postoperative axial T2-weighted image showing no evidence of residual cavernous malformation.

crosoft SPSS v21.0 statistical package. The $\alpha$ criterion of 0.05 was used to define statistical significance. The 1-way ANOVA test was used to evaluate for statistically significant variability in the mean values of surgically relevant distances between 3 groups (right-handed, left-handed, and ambidextrous). The paired t-test was used to compare the mean distances on the left side versus the right side within each group.

\section{Anatomical Dissections}

Four fresh human head specimens were prepared for dissection at the Surgical Neuroanatomy Laboratory of the Department of Neurological Surgery at the University of Pittsburgh School of Medicine. The research was approved by the Committee for Oversight of Research Involving the Dead (CORID) at the University of Pittsburgh. The specimens were only used for illustrations of the relevant anatomy; no measurements were performed in these specimens for use in our statistical analyses.

\section{Institutional Review Board, Consent, and Sources of Funding}

The study was performed under an approved University of Pittsburgh Institutional Review Board protocol. Please note that the MRI scans and the acquisition of the original diffusion data were performed under separate institutional review board protocols overseeing the Washington University-University of Minnesota HCP consortium research practices. The illustrative case was presented with the patient's consent. The study was made possible with funds provided by the Pittsburgh Brain Institute.

\section{Illustrative Case}

A 60-year-old man who was receiving anticoagulation therapy with warfarin because of a mechanical mitral valve presented with recurrent bleeding events from a known pontine cavernous malformation. On physical examination he had a partial left CN VI palsy and partial ptosis, left facial hypesthesia in all trigeminal distributions, a left central facial weakness, and left-sided dysmetria, but was intact in motor strength otherwise. He also had debilitating vertigo. Steroids did not improve his symptoms. The patient's presenting MRI is shown in Fig. 2 (of note, he had a pacemaker, which was compatible with the clinical 1.5-T MRI unit, but was a contraindication for 3-T HDFT MRI). Given the recurrent and debilitating symptoms, and the need for lifelong anticoagulation, surgical evacuation was recommended.

Because the lesion significantly extended both above and below the level of the trigeminal nerve, the epitrigeminal approach was thought to be more appropriate. The subtemporal transtentorial surgical corridor provides an angle of attack that approximates the long axis of the brainstem, allowing for rostrocaudal flexibility. Thus, working medially to the trigeminal nerve and posterior to the CSTs, the epitrigeminal approach provides access to the ventrolateral pons that extends both above and below the level of the trigeminal nerve. The key steps of the procedure are summarized in Fig. 3 and Video 1.

VIDEO 1. Clip showing the key steps of the epitrigeminal approach in a case of a brainstem cavernous malformation. Copyright

Georgios A. Zenonos. Published with permission. Click here to view.

The patient was discharged after a short, uneventful hospital course and resumption of his anticoagulation therapy. At 4 months of follow-up, there was no evidence of residual cavernous malformation on imaging (Fig. 2). The patient's vertigo, ataxia, and facial motor function had recovered completely. His ptosis, CN VI palsy, and trigeminal distribution numbness had improved. There was no evidence of $\mathrm{CN} \mathrm{V}$ motor weakness.

\section{Results of HDFT Anatomical Study}

The surgically relevant distances evaluated by our HDFT study are analyzed in Table 1 . The direct and vertical distances of the CSTs from the trigeminal entry zones on each side were evaluated separately for right-handed, left-handed, and ambidextrous individuals. When comparing the mean distances with ANOVA across these 3 groups for the left and right side separately, we did not find a statistically significant variability. When evaluating the whole group, the average direct distance was $12.6 \mathrm{~mm}$ (range 8.7-17 mm). The average vertical distance was $3.6 \mathrm{~mm}$ (range -2.3 to $8.7 \mathrm{~mm}$ ). The negative values in the vertical distances were observed because of 1 patient in whom the posterolateral margin of the CST was located dorsal to the intertrigeminal line. When excluding this patient, the vertical distances ranged from $0 \mathrm{~mm}$ to $8.7 \mathrm{~mm}$. When comparing the direct and vertical distances on the left versus the right side within each group (right-handed, left-handed, and ambidextrous) by using the paired t-test, we found no statistically significant differences. Interestingly, however, 

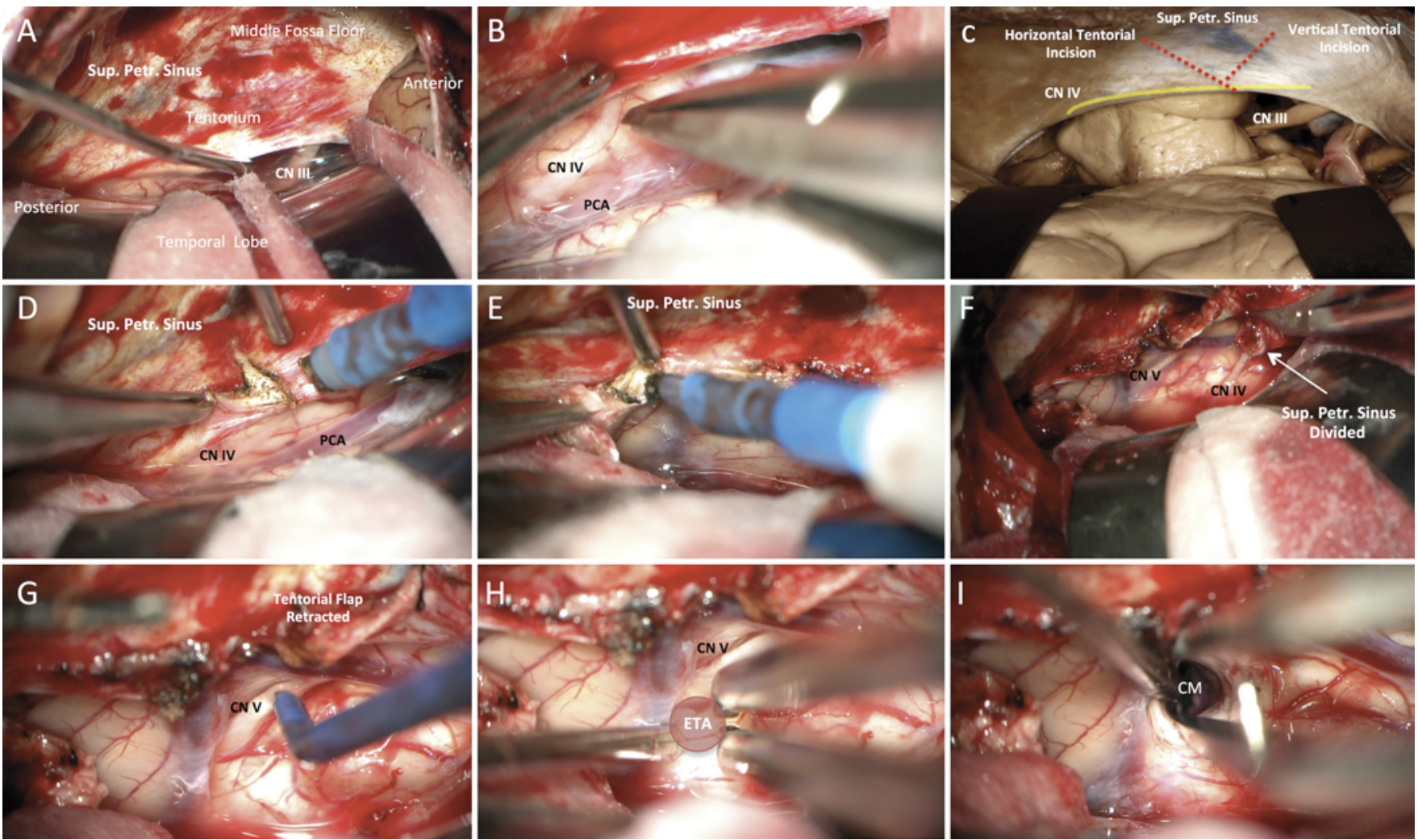

FIG. 3. Key surgical steps for the epitrigeminal approach to the brainstem via a left subtemporal transtentorial corridor. A: After elevation of the temporal lobe, CN III and the superior petrosal sinus (Sup. Petr. Sinus) are visualized. B: Arachnoid dissection in the ambient cistern brings CN IV and the posterior cerebral artery (PCA) into view. C: Tentorial cuts: a horizontal cut in parallel to the superior petrosal sinus starting at the point where CN IV crosses the tentorium, and a vertical cut across the superior petrosal sinus. D-F: The steps of the tentorial cuts in our case. G: The tentorial leaflet is reflected, revealing CN V. The motor fibers of $\mathrm{CN} V$ are stimulated. H: The area just above the trigeminal entry zone, the epitrigeminal area (ETA) is coagulated with bipolar electrocautery. I: After a short parenchymal dissection, the cavernous malformation (CM) is exposed and resected in a piecemeal fashion.

there was a statistically nonsignificant trend for increased vertical and direct distances on the left side in each group.

\section{Discussion}

Dorsal pontine lesions have been approached through safe entry zones in the rhomboid fossa. ${ }^{3-6,14,15,17}$ For ventral or ventrolateral lesions, a number of other safe entry zones have been described. ${ }^{3-6,9,11,14,15,17} \mathrm{~A}$ common denominator for all of these safe entry zones to the ventrolateral pons is that they are designed to avoid injury to 2 main structures: the CSTs and the trigeminal nerve. Before we discuss the relevant approaches to the ventrolateral pons, it's useful to briefly review some surgically relevant points of pontine anatomy.

\section{Surgically Relevant Pontine Anatomy}

The gross external anatomy of the pons is presented in Fig. 4A. A critical anatomical relationship for the surgeon is the projection of the corticobulbar and corticospinal fibers on the ventral surface of the pons (Fig. 4B-D). These fibers have no clear surface landmark in the pons, and therefore their location has to be extrapolated from external landmarks of the midbrain and medulla. The lateral extent of the motor fibers can be approximated by an imaginary line extending from the lateral aspect of the crus cerebri in the midbrain to the lateral aspect of the pyramid in the medulla. ${ }^{15}$ Of note, only the middle two-thirds of the cerebral peduncles in the midbrain correspond to the corticobulbar and corticospinal motor fibers. These pyramidal fibers are surrounded by corticopontine fibers, which are further subdivided into frontopontine and temporopontine fibers, lying medially and laterally, respectively, from the pyramidal fibers in each crus cerebri. It is worth noting that the diameter of the motor tracts in the ventral pons is larger toward the midbrain and smaller toward the medulla, averaging approximately $12.2 \mathrm{~mm}, 8.4 \mathrm{~mm}$, and 5.6 $\mathrm{mm}$ in the upper, middle, and lower thirds, respectively. ${ }^{15}$

Intraoperatively, the full extent of the cerebral peduncles and the pyramids is often not visible, and therefore it is frequently not feasible to gauge the position of the pyramidal fibers based on these landmarks. For this reason, it is useful to know the approximate distances of the corticospinal fibers from more accessible landmarks, such as the entry zone of the trigeminal nerve. In one study, the distance between the pyramidal fibers and $\mathrm{CN} \mathrm{V}$ ranged from $3.8 \mathrm{~mm}$ to $5.6 \mathrm{~mm}$ (average $4.64 \mathrm{~mm}$ ). ${ }^{15} \mathrm{In}$ another report, this distance was estimated to average 9.2 
TABLE 1. Surgically relevant anatomical variability of the CSTs relative to the epitrigeminal entry zone

\begin{tabular}{|c|c|c|c|c|c|c|c|}
\hline \multirow[b]{3}{*}{ Measurement } & \multicolumn{6}{|c|}{ Handedness } & \multirow{3}{*}{$\begin{array}{c}\text { p Value } \\
\text { (ANOVA) }\end{array}$} \\
\hline & \multicolumn{2}{|c|}{ Rt-Handed, $n=61$} & \multicolumn{2}{|c|}{ Lt-Handed, $\mathrm{n}=10$} & \multicolumn{2}{|c|}{ Ambidextrous, $n=6$} & \\
\hline & Mean, SD (mm) & Range (mm) & Mean, SD (mm) & Range (mm) & Mean, SD (mm) & Range (mm) & \\
\hline \multicolumn{8}{|l|}{ Vertical distance } \\
\hline Rt & $3.46,1.96$ & -1.32 to 7.46 & $3.99,1.48$ & 1.32 to 6.29 & $2.83,1.43$ & 1.29 to 5.00 & 0.481 \\
\hline Lt & $3.78,1.86$ & -2.34 to 8.16 & $4.37,1.96$ & 1.25 to 8.71 & $3.04,1.87$ & 1.00 to 5.00 & 0.387 \\
\hline Paired t-test $†$ & $p=0.071$ & & $p=0.494$ & & $p=0.478$ & & \\
\hline Intertrigeminal distance & $39.1,2.5$ & 33.7 to 45.0 & $38.9,2.4$ & 36.3 to 42.6 & $38.8,2.4$ & 35.0 to 45.3 & 0.927 \\
\hline \multicolumn{8}{|l|}{ Direct distance } \\
\hline Rt & $12.48,1.76$ & 9.03 to 17.00 & $11.7,1.31$ & 10.10 to 14.00 & $12.16,1.82$ & 9.57 to 14.20 & 0.400 \\
\hline $\mathrm{Lt}$ & $12.77,1.88$ & 8.68 to 16.3 & $12.79,1.98$ & 9.00 to 15.70 & $12.72,1.65$ & 11.10 to 14.90 & 0.997 \\
\hline Paired t-test $\dagger$ & $p=0.229$ & & $p=0.122$ & & $p=0.322$ & & \\
\hline
\end{tabular}

See Fig. 1 for definitions of the measurements. Negative values in the vertical distance suggest that the CSTs extend dorsal to the intertrigeminal line.

* ANOVA p values compare the mean distances across the 3 groups (right-handed, left-handed, and ambidextrous).

$\dagger$ Paired t-test $p$ values compare the mean left and right distances within each group.

$\mathrm{mm}$, whereas the distance from the pyramidal tracts to the nerve root exit zone of the facial nerve was found to average $8 \mathrm{~mm} .{ }^{17}$ Both of the aforementioned studies were based on anatomical dissections, which are subject to several limitations. One such limitation is the difficulty in evaluating the full course of the tracts, making it virtually impossible to differentiate among parallel tracts. One such example would be the differentiation between the frontopontine, corticobulbar, corticospinal, and temporopontine tracts in the pons. Conversely, one advantage of the HDFT studies is that the full extent of a tract can be more reliably defined. In our HDFT anatomical study, the average direct distance of the CSTs from the posterior- and superiormost point of the trigeminal entry zone was $12.6 \mathrm{~mm}$, ranging from $8.7 \mathrm{~mm}$ to $17 \mathrm{~mm}$. However, the average vertical distance was $3.6 \mathrm{~mm}$, ranging from $-2.3 \mathrm{~mm}$ to $8.7 \mathrm{~mm}$. This shorter vertical distance translates into a narrower operative corridor. As mentioned in the Results section above, in 1 patient the CSTs were extending dorsal to the trigeminal zones, minimizing the safe entry zone of most of the approaches analyzed below. The internal anatomy of the pons is analyzed in Fig. 4E-H.

\section{The Peritrigeminal, Supratrigeminal, Lateral Pontine, and Lateral Transpeduncular Approaches to the Ventrolateral Pons}

The approaches described below are summarized in Table 2 and Figs. 5 and 6.

\section{Peritrigeminal Approach}

The most commonly cited safe entry zone to the ventrolateral pons for lesions ventral to the plane of the medial lemniscus is the peritrigeminal zone (Fig. 5A). ${ }^{1-5,10,14,15,17}$ This area is usually exposed via a retrosigmoid, a presigmoid, or an extended middle fossa approach (Kawase's), and roughly stretches from the trigeminal to the facial nerves. ${ }^{1-5,10,14,15,17}$ The peritrigeminal zone can be conceptualized as a triangular area, which is defined medially by the estimated lateral border of the CSTs (extending from the lateral aspect of the crura cerebri to the lateral aspect of the pyramids), inferiorly by the pontomedullary sulcus, and laterally by a line passing from the lateral aspect of the crus cerebri to the point of entry of the trigeminal nerve, intersecting the pontomedullary sulcus inferiorly. ${ }^{15}$ The floor of this peritrigeminal area is composed of transverse pontine fibers, which course posteriorly and slightly caudally in a rather oblique trajectory.

Whether using a vertical or horizontal pial incision, the critical concern is to avoid injury to the underlying CSTs which, as mentioned earlier, can lie very close to the trigeminal entry zone. ${ }^{1-5,10,14,15,17}$ In addition, structures that the surgeon should be aware of and avoid are the trigeminal spinal tract, the trigeminal motor and sensory nuclei located approximately $10 \mathrm{~mm}$ dorsomedial to the trigeminal entry zone, ${ }^{17}$ and the intrapontine segments of the facial and abducent nerves. A relative limitation of this approach is that lesions with significant extension above the level of $\mathrm{CN} \mathrm{V}$ cannot be approached without rostral displacement of the intrapontine segment of the trigeminal nerve, which increases the risk of trigeminal neuropathy. Notably, the peritrigeminal zone is equivalent to the lateral pontine zone, referring to the area stretching between CNs V and VII, at the junction of the middle cerebellar peduncle with the pons (the latter corresponding roughly to the trigeminal nerve entry zone)., ${ }^{1,4}$

\section{Lateral Transpeduncular Approach}

The lateral transpeduncular approach is an alternative to the previously described peritrigeminal approach, providing access to lesions with more rostral and posterior extension in the ventrolateral pons (Fig. 5B). ${ }^{11}$ This approach deviates to some extent from the classic "2-point rule" originally described by the senior author of the same report, because it follows a somewhat longer intraparenchymal trajectory to reach the lesion. After exposing the lateral pons via a retrosigmoid approach, the authors describe breaching the pia on the lateral aspect of the middle cerebellar peduncle, close to where the peduncle meets the cerebellar hemisphere. The pial incision is made caudal to the emergence of $\mathrm{CN} \mathrm{V}$, and rostral to the pontomedul- 

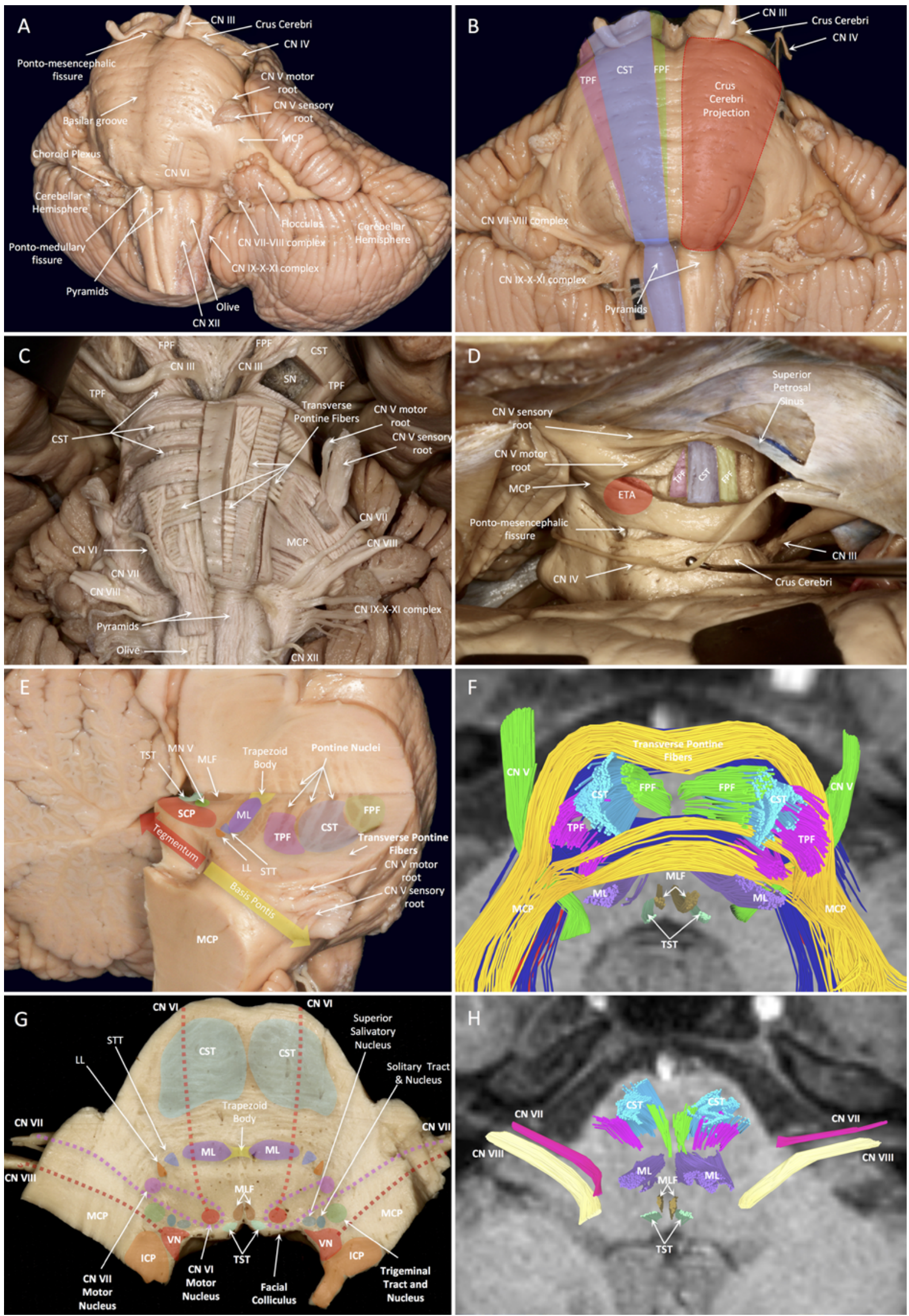

FIG. 4. Pontine anatomy. A: Gross anatomy of the pons. FIG. 4. (continued) $\rightarrow$ 
FIG. 4. B: Projection of descending fibers of the crus cerebri in the pons. On the left, the corticospinal fibers (or CSTs) are seen occupying the middle two-thirds of the descending fibers. These fibers are surrounded by frontopontine fibers medially, and temporopontine fibers laterally. C: Partial white matter dissection in the pons showing the descending CST intersecting the transverse pontine fibers. D: View from a subtemporal-transtentorial surgical corridor showing the relationship of the descending tracts with the epitrigeminal area. After removing the superficial transverse pontine fibers we see, from rostral to caudal (right to left), the frontopontine, corticospinal, and temporopontine fibers, respectively. E-H: The trapezoid body divides the pons into the tegmentum and basis pontis. The paired medial lemnisci lie in the anterior tegmentum, whereas the spinal and lateral lemnisci lie just lateral and slightly posterior to the medial lemnisci. In the basis pontis, small condensations of gray matter form the pontine nuclei, which give rise to the transverse pontine fibers. E: Internal pontine anatomy at the level just above the trigeminal entry zone. F: Corresponding HDFT image showing the fiber tracts at the same level. G: Internal pontine anatomy at the level just above the pontomedullary fissure. H: Corresponding HDFT image showing the fiber tracts at the same level. ETA = epitrigeminal area; FPF = frontopontine fibers; ICP = inferior cerebellar peduncle; $L L=$ lateral lemniscus; $M C P=$ middle cerebellar peduncle; $M L$ = medial lemniscus; MLF = medial longitudinal fasciculus; MN V = motor nucleus of CN V; SCP = superior cerebellar peduncle; SN = substantia nigra; STT = spinothalamic tract; TPF = temporopontine fibers; TST = tectospinal tract; VN = vestibular nuclei.

lary sulcus. Using image guidance, the authors describe a rostro-ventro-medial trajectory, which courses above the intrapontine segment of the trigeminal nerve to reach the lesion. Because of this "above the trigeminal" course, the same group refers to this approach in a later report as a "supra-trigeminal" approach, ${ }^{4}$ which should not be confused with the supratrigeminal approach described below. The authors report good results and minimal morbidity associated with transgression of the middle cerebellar peduncle. Although this approach is suitable for lesions extending above the level of the $\mathrm{CN} \mathrm{V}$, addressing pathology with more caudal extension would require downward displacement of the intrapontine segment of the trigeminal nerve, increasing the risk for trigeminal neuropathy. Furthermore, this approach is heavily dependent on image guidance to achieve a supratrigeminal corridor, and therefore the margin for error is small.

\section{Supratrigeminal Approach}

The so-called supratrigeminal approach, described by Cavalheiro et al., 5 is an approach mainly to the rostroventral pons (Fig. 5C). This approach refers to transgression of a vertical area extending below the emergence of CN III in the midbrain, and extending approximately $4 \mathrm{~mm}$ inferiorly to a level just above the axial plane of the trigeminal nerve entry zone. The anatomical basis for this approach is that the very medial pial incision avoids the true pyramidal fibers (the corticobulbar and corticospinal motor fibers), which occupy the middle two-thirds of the cerebral peduncles, affecting only the medial frontopontine fibers. This approach has been described mainly through an exposure provided by an orbitozygomatic craniotomy, dissection of the interpeduncular and prepontine cisterns, and division of the free edge of the tentorium..$^{5}$ This approach again provides a good ventral access for biopsy sampling of lesions in the rostral ventrolateral pons, but the long surgical corridor and the proximity of the CSTs make resections of lesions very challenging. In addition, this approach may require manipulation of the basilar artery in the basilar groove, which increases the risk of vascular injury or stroke.

\section{The Epitrigeminal Approach-Relevant Anatomy and Surgical Considerations}

Although versions of so-called "above the trigeminal nerve" approaches have been described in the literature, all of them referred to as supratrigeminal approaches, ${ }^{4,5,11}$ these differ substantially from the approach we analyze below. To avoid confusion with prior reports of supratrigeminal approaches we refer to the described approach as epitrigeminal (Fig. 5D).

In our case, division of the tentorium provided adequate

TABLE 2. Summary of safe entry zones to the ventrolateral pons

\begin{tabular}{|c|c|c|c|c|}
\hline $\begin{array}{l}\text { Safe Entry Zone } \\
\text { (alternative } \\
\text { names) }\end{array}$ & $\begin{array}{l}\text { Brief Anatomical } \\
\text { Description }\end{array}$ & $\begin{array}{l}\text { Surgical } \\
\text { Corridor(s) }\end{array}$ & Advantages & Disadvantages \\
\hline $\begin{array}{l}\text { Peritrigeminal } \\
\quad \text { (lateral pon- } \\
\text { tine })^{1-5,10,14,15,17}\end{array}$ & $\begin{array}{l}\text { Triangular area stretching from } \\
\text { CN V to CNs VII \& VIII }\end{array}$ & $\begin{array}{l}\text { Retrosigmoid, presig- } \\
\text { moid, extended middle } \\
\text { fossa (Kawase's) }\end{array}$ & $\begin{array}{l}\text { No temporal lobe retraction, } \\
\text { good exposure for caudal } \\
\text { ventrolateral pontine lesions }\end{array}$ & $\begin{array}{l}\text { Not suitable for lesions significantly } \\
\text { extending above the level of } \mathrm{CN} \\
\mathrm{V}, \text { or those w/o lateral extension }\end{array}$ \\
\hline $\begin{array}{l}\text { Lateral transpe- } \\
\text { duncular } \\
\text { (supratrigemi- } \\
\text { nal) })^{4,11}\end{array}$ & $\begin{array}{l}\text { Entry zone in lateral brachium } \\
\text { pontis, w/ an intraparenchymal } \\
\text { trajectory coursing above the } \\
\text { intrapontine segment of } \mathrm{CN} \mathrm{V}\end{array}$ & Retrosigmoid craniotomy & $\begin{array}{l}\text { No temporal lobe retraction, } \\
\text { good exposure for rostral } \\
\text { ventrolateral pontine lesions }\end{array}$ & $\begin{array}{l}\text { Not suitable for lesions w/ significant } \\
\text { extension below the level of } \mathrm{CN} \\
\mathrm{V} \text {, or those w/o lateral extension; } \\
\text { relies heavily on image guidance }\end{array}$ \\
\hline Supratrigeminal ${ }^{5}$ & $\begin{array}{l}\text { Area extending approximately } \\
4 \text { mm inferiorly from CN III, } \\
\text { midline, or paramidline }\end{array}$ & $\begin{array}{l}\text { Cranio-orbitozygomatic } \\
\text { craniotomy w/ dissec- } \\
\text { tion of the membrane } \\
\text { of Liliequist }\end{array}$ & $\begin{array}{l}\text { No temporal lobe retraction, } \\
\text { more suitable for rostroven- } \\
\text { tral lesions in the pons w/o } \\
\text { significant lateral extension }\end{array}$ & $\begin{array}{l}\text { Extensive craniotomy, long \& } \\
\text { narrow surgical corridor; limited } \\
\text { exposure other than the midline } \\
\text { rostroventral pons }\end{array}$ \\
\hline Epitrigeminal & $\begin{array}{l}\text { Area just above the trigeminal } \\
\text { entry zone }\end{array}$ & $\begin{array}{l}\text { Subtemporal transtento- } \\
\text { rial approach }\end{array}$ & $\begin{array}{l}\text { Versatile for pontine lesions w/ } \\
\text { either significant rostral or } \\
\text { caudal extensions }\end{array}$ & $\begin{array}{l}\text { Temporal lobe retraction; probably } \\
\text { not ideal for lesions w/o lateral } \\
\text { extension }\end{array}$ \\
\hline
\end{tabular}



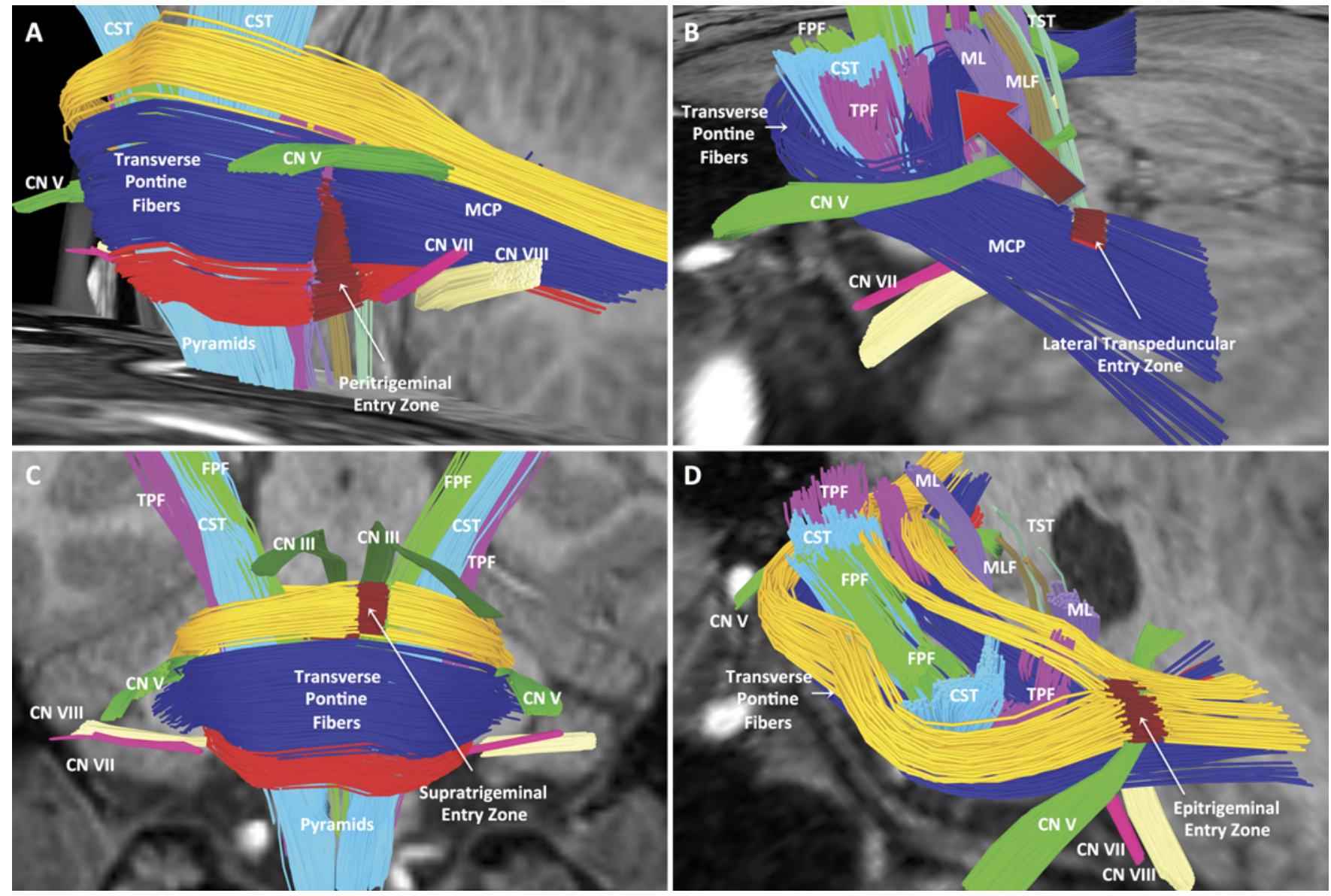

FIG. 5. High-definition fiber tractography studies showing the safe entry zones to the ventrolateral pons. The safe entry zones are shaded in red. A: The peritrigeminal entry zone extends from the trigeminal entry zone above, to the level just anterior to the facial nerve below. B: The lateral transpeduncular approach starts by transgression of the lateral fibers of the brachium pontis. Subsequently, as shown by the red arrow, following a rostro-ventro-medial course within the middle cerebellar peduncle, the surgical corridor passes above the intrapontine segment of the trigeminal nerve to reach the rostral ventrolateral pons. C: The supratrigeminal safe entry zone is located right beneath the oculomotor nerve and disrupts only some of the frontopontine fibers. D: The epitrigeminal entry zone is located just above the posterior margin of the trigeminal entry zone. See Fig. 4 legend for definitions of abbreviations.

access to the area above the trigeminal nerve entry zone. Notably, although the subtemporal transtentorial approach without a petrosectomy is thought not to provide access to lesions below the level of the trigeminal entry zone, this concept refers to extraaxial lesions at the surface of the brainstem. However, due to the rostrocaudal trajectory, as well as the conical shape of the surgical corridor (increasing the exposed area the farther one proceeds from the entry point), the caudal extent of the exposure expands as we advance toward the midline. In effect, deep-seated lesions within the brainstem parenchyma can be accessed despite their caudal extension below the trigeminal nerve (Fig. 6). This was true in our case, in which the cavernous malformation extended down to the pontomedullary fissure.

It is also useful to discuss the intrinsic brainstem anatomy relevant to this surgical corridor (Figs. 4E-H and 5D). First, the descending corticospinal and corticobulbar fibers are located just medial and anterior to the trigeminal nerve entry zone at a distance that can be as short as 3.8 $\mathrm{mm} . .^{15}$ Notably, as found in our study, on rare occasions the CSTs may extend behind the trigeminal entry zone, minimizing the surgical corridor. Second, the intrapontine segment of $\mathrm{CN} \mathrm{V}$ courses posteromedially to end in the principal and motor trigeminal nuclei at a distance of approximately $10.1 \mathrm{~mm}$ from the trigeminal nerve entry zone. ${ }^{17}$ The transversely oriented medial lemniscus lies slightly anteroventral to these nuclei. Between the medial lemnisci and the trigeminal nuclei lie the anterolateral spinothalamic tracts, the trigeminothalamic tracts, and others such as the rubrospinal tract and the central tegmental tract. It is also important to remember that although the nuclei of CNs VI and VII lie inferiorly, they are still located above the plane of the pontomedullary fissure, with the intrapontine segment of these nerves following an anteroinferior course before exiting from the pontomedullary fissure. In effect, a surgical corridor just above the trigeminal entry zone usually avoids injury to all the aforementioned structures unless the dissection is carried out significantly ventrally or dorsally. Of course the normal anatomy can be significantly distorted by the pathological entity, and this has to be accounted for in the surgical planning. 


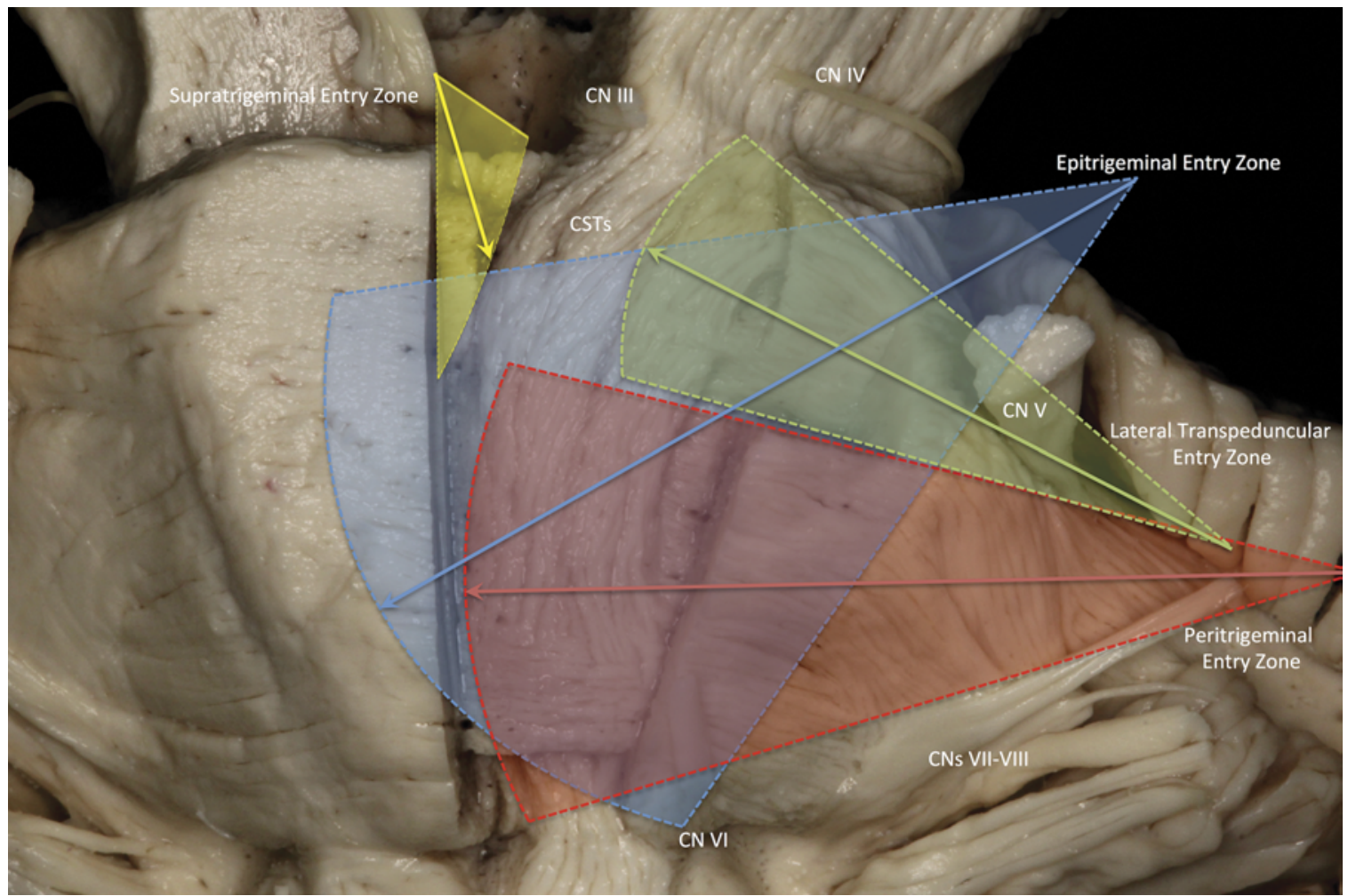

FIG. 6. Angle of attack of safe entry zones and associated surgical corridors to the ventrolateral pons. The epitrigeminal approach through a subtemporal transtentorial surgical corridor (light blue) has an angle of attack that better approximates the long axis of the brainstem, providing good rostrocaudal flexibility. The peritrigeminal approach (light red), and the lateral transpeduncular approaches (light green) through a retrosigmoid surgical corridor are best suited for lesions extending below and above the level of the trigeminal nerve, respectively. The supratrigeminal entry zone has a long surgical corridor provided by an orbitozygomatic craniotomy, and is significantly limited by the proximity to the CSTs.

\section{Comparison of the Epitrigeminal Approach With the Other Described Approaches to the Ventrolateral Pons}

The epitrigeminal approach has several points that differ from the aforementioned approaches to the ventrolateral pons, which are summarized in Table 2 and Figs. 5 and 6. One possible advantage of this approach is that is can be very versatile in the rostrocaudal axis, providing access to lesions significantly extending both above and below the trigeminal nerve. The peritrigeminal approach and the lateral transpeduncular approach are best suited for lesions extending below and above the level of the trigeminal nerve, respectively (Fig. 6). Of course, retraction of the intrapontine segment of the trigeminal nerve rostrally (peritrigeminal), caudally (lateral transpeduncular), or laterally (epitrigeminal) can enhance the surgical corridor of all 3 of these approaches, although with an increased risk of trigeminal neuropathy. The supratrigeminal entry zone has a long surgical corridor and is significantly limited by the proximity to the CSTs, making it more appropriate for biopsies as opposed to resections of pontine lesions. On the other hand, one of the relative downsides of the epitrigeminal approach is that the subtemporal corridor requires some temporal lobe retraction. Placement of a lumbar drain, however, usually provides adequate brain relaxation so that only gentle retraction is required, and thus retraction injury is fairly uncommon. Furthermore, the subtemporal-transtentorial surgical corridor is slightly longer than the one provided by a retrosigmoid craniotomy, and variations of the vein of Labbé may limit the amount of temporal lobe retraction that can be achieved, and therefore limit the rostrocaudal angle of attack.

\section{Limitations of the Study}

Despite having used a tracking method that achieved the best valid connection with more than $90 \%$ accuracy when examined in an open competition, ${ }^{12}$ no fiber-tracking method is without flaws. Thus, imperfections may exist in seed placement, track propagation, and termination location. Given that no gold standard exists against which HDFT can be validated, the exact accuracy for specific tracts is not known, and therefore accuracy metrics cannot be incorporated in our statistical analyses. Furthermore, although large differences in the surgically relevant distances were not found, the low number of left-handed and ambidextrous subjects studied may limit our statistical power in detecting small differences. In addition, more clinical experience is required to solidify the safety and effectiveness of the epitrigeminal approach for intrinsic brainstem lesions.

\section{Conclusions}

The epitrigeminal approach seems to be safe and effective for treating intrinsic ventrolateral pontine pathological 
entities. A possible advantage of this approach is increased versatility in the rostrocaudal axis, providing access both above and below the trigeminal nerve. Familiarity with the subtemporal transtentorial approach, and the reliable surgical landmark of the trigeminal nerve entry zone, should make this a straightforward approach.

\section{Acknowledgments}

This study was funded by the University of Pittsburgh Brain Institute.

\section{References}

1. Baghai P, Vries JK, Bechtel PC: Retromastoid approach for biopsy of brain stem tumors. Neurosurgery 10:574-579, 1982

2. Bertalanffy H, Benes L, Miyazawa T, Alberti O, Siegel AM, Sure U: Cerebral cavernomas in the adult. Review of the literature and analysis of 72 surgically treated patients. Neurosurg Rev 25:1-55, 2002

3. Cantore G, Missori P, Santoro A: Cavernous angiomas of the brain stem. Intra-axial anatomical pitfalls and surgical strategies. Surg Neurol 52:84-94, 1999

4. Cavalcanti DD, Preul MC, Kalani MY, Spetzler RF: Microsurgical anatomy of safe entry zones to the brainstem. J Neurosurg 124:1359-1376, 2016

5. Cavalheiro S, Yagmurlu K, da Costa MD, Nicácio JM, Rodrigues TP, Chaddad-Neto F, et al: Surgical approaches for brainstem tumors in pediatric patients. Childs Nerv Syst 31:1815-1840, 2015

6. Duckworth EA: Modern management of brainstem cavernous malformations. Neurol Clin 28:887-898, 2010

7. Faraji AH, Abhinav K, Jarbo K, Yeh FC, Shin SS, Pathak $\mathrm{S}$, et al: Longitudinal evaluation of corticospinal tract in patients with resected brainstem cavernous malformations using high-definition fiber tractography and diffusion connectometry analysis: preliminary experience. J Neurosurg 123:1133-1144, 2015

8. Fernandes-Cabral DT, Zenonos GA, Hamilton RL, Panesar SS, Fernandez-Miranda JC: High-definition fiber tractography in the evaluation and surgical planning of Lhermitte-Duclos disease: a case report. World Neurosurg 92:587.e9-587. e13, 2016

9. Gross BA, Dunn IF, Du R, Al-Mefty O: Petrosal approaches to brainstem cavernous malformations. Neurosurg Focus 33(2):E10, 2012

10. Hauck EF, Barnett SL, White JA, Samson D: The presigmoid approach to anterolateral pontine cavernomas. Clinical article. J Neurosurg 113:701-708, 2010

11. Hebb MO, Spetzler RF: Lateral transpeduncular approach to intrinsic lesions of the rostral pons. Neurosurgery 66 (3 Suppl Operative):26-29, 2010

12. Maier-Hein K, Neher P, Houde JC, Cote MA, Garyfallidis E, Zhong J, et al: Tractography-based connectomes are dominated by false-positive connections. bioRxiv [epub ahead of print], 2016
13. Meola A, Yeh FC, Fellows-Mayle W, Weed J, FernandezMiranda JC: Human connectome-based tractographic atlas of the brainstem connections and surgical approaches. Neurosurgery 79:437-455, 2016

14. Petr O, Lanzino G: Brainstem cavernous malformations. J Neurosurg Sci 59:271-282, 2015

15. Recalde RJ, Figueiredo EG, de Oliveira E: Microsurgical anatomy of the safe entry zones on the anterolateral brainstem related to surgical approaches to cavernous malformations. Neurosurgery 62 (3 Suppl 1):9-17, 2008

16. Sotiropoulos SN, Jbabdi S, Xu J, Andersson JL, Moeller S, Auerbach EJ, et al: Advances in diffusion MRI acquisition and processing in the Human Connectome Project. Neuroimage 80:125-143, 2013

17. Yagmurlu K, Rhoton AL Jr, Tanriover N, Bennett JA: Threedimensional microsurgical anatomy and the safe entry zones of the brainstem. Neurosurgery 10 (Suppl 4):602-620, 2014

18. Yeh FC, Verstynen TD, Wang Y, Fernández-Miranda JC, Tseng WY: Deterministic diffusion fiber tracking improved by quantitative anisotropy. PLoS One 8:e80713, 2013

19. Yeh FC, Wedeen VJ, Tseng WY: Generalized q-sampling imaging. IEEE Trans Med Imaging 29:1626-1635, 2010

20. Yoshino M, Abhinav K, Yeh FC, Panesar S, Fernandes D, Pathak S, et al: Visualization of cranial nerves using highdefinition fiber tractography. Neurosurgery 79:146-165, 2016

\section{Disclosures}

The authors report no conflict of interest concerning the materials or methods used in this study or the findings specified in this paper.

\section{Author Contributions}

Conception and design: Zenonos. Acquisition of data: Zenonos, Fernandes-Cabral, Nunez, Lieber. Analysis and interpretation of data: Zenonos, Fernandes-Cabral. Drafting the article: Zenonos. Critically revising the article: Zenonos, Fernandes-Cabral, Friedlander. Reviewed submitted version of manuscript: Zenonos, Fernandes-Cabral, Fernandez-Miranda, Friedlander. Approved the final version of the manuscript on behalf of all authors: Zenonos. Statistical analysis: Zenonos. Administrative/technical/material support: Zenonos, Fernandes-Cabral. Study supervision: Zenonos, Friedlander. Anatomical dissections: Nunez, Lieber.

\section{Supplemental Information \\ Videos}

Video 1. https://vimeo.com/212087182.

\section{Correspondence}

Georgios Zenonos, Department of Neurosurgery, Presbyterian Hospital, 200 Lothrop St., Ste. B400, Pittsburgh, PA 15213. email: zenonosg@upmc.edu. 